\title{
Longitudinal assessment of antibiotic resistance gene profiles in gut microbiomes of infants at risk of eczema
}

Evelyn Xiu Ling Loo ${ }^{1,2+}$, Amanda Zain ${ }^{2,3 \dagger}$, Gaik Chin Yap², Rikky W. Purbojati ${ }^{4}$, Daniela I. Drautz-Moses ${ }^{4}$, Yan Qing Koh ${ }^{4}$, Yap Seng Chong ${ }^{1,5}$, Kok Hian Tan ${ }^{6}$, Peter D. Gluckman ${ }^{1,7}$, Fabian Yap ${ }^{8}$, Johan Gunnar Eriksson ${ }^{1,5}$, Elizabeth Tham ${ }^{2,3}$, Lynette Pei-chi Shek ${ }^{1,2,3}$, Staffan Kjelleberg ${ }^{4}$, Stephan C. Schuster ${ }^{4}$, Ritu Banerjee ${ }^{9}$ and Bee Wah Lee 2* $^{*}$

\begin{abstract}
Background: While there is increasing knowledge about the gut microbiome, the factors influencing and the significance of the gut resistome are still not well understood. Infant gut commensals risk transferring multidrugresistant antibiotic resistance genes (ARGs) to pathogenic bacteria. The rapid spread of multidrug-resistant pathogenic bacteria is a worldwide public health concern. Better understanding of the naïve infant gut resistome may build the evidence base for antimicrobial stewardship in both humans and in the food industry. Given the high carriage rate of extended spectrum beta-lactamase (ESBL)-producing Enterobacteriaceae in Asia, we aimed to evaluate community prevalence, dynamics, and longitudinal changes in antibiotic resistance gene (ARG) profiles and prevalence of ESBLproducing E. coli and K. pneumoniae in the intestinal microbiome of infants participating in the Growing Up in Singapore Towards Healthy Outcomes (GUSTO) study, a longitudinal cohort study of pregnant women and their infants.
\end{abstract}

Methods: We analysed ARGs in the first year of life among 75 infants at risk of eczema who had stool samples collected at multiple timepoints using metagenomics.

Results: The mean number of ARGs per infant increased with age. The most common ARGs identified confer resistance to aminoglycoside, beta-lactam, macrolide and tetracycline antibiotics; all infants harboured these antibiotic resistance genes at some point in the first year of life. Few ARGs persisted throughout the first year of life. Beta-lactam resistant Escherichia coli and Klebsiella pneumoniae were detected in 4 (5.3\%) and 32 (42.7\%) of subjects respectively.

Conclusion: In this longitudinal cohort study of infants living in a region with high endemic antibacterial resistance, we demonstrate that majority of the infants harboured several antibiotic resistance genes in their gut and showed that the infant gut resistome is diverse and dynamic over the first year of life.

Keywords: Antibiotic resistance genes, Resistome, Infancy, Birth cohort, ESBL producing Enterobacteriaceae

\footnotetext{
* Correspondence: Paeleebw@nus.edu.sg

${ }^{\dagger}$ Evelyn Xiu Ling Loo and Amanda Zain are co-first authors.

${ }^{2}$ Department of Paediatrics, Yong Loo Lin School of Medicine, National University of Singapore, Singapore, Singapore

Full list of author information is available at the end of the article
} 


\section{Background}

The rapid spread of multidrug-resistant pathogenic bacteria that are no longer susceptible to treatment with common antibiotics is a global public health concern. The lack of efficacy of currently available antibiotics and the paucity of new antibiotic development may potentially result in a situation akin to the pre-antibiotic era where minor infections may become life-threatening conditions [1-3].

The human gastrointestinal tract hosts a diversity of microbiota which are responsible for important functions such as stimulation of intestinal angiogenesis, protection against cell injury and regulation of fat storage. However, the gut microbiota is a reservoir for antibiotic resistance genes (ARGs). ARGs have been identified in the intestinal microbiomes from adults as well as infants as early as the first month of life [4-9]. Alarmingly, ARGs in commensal human gut microbiota can be transferred to pathogenic strains of bacteria that can cause disease [10].

There is significant geographic variation in the prevalence and type of ARGs in microbiota, implying that there may be environmental and lifestyle factors affecting the prevalence of ARGs. Hu and colleagues reported regional differences in ARG abundance; Chinese individuals have the greatest number of ARGs, followed by Danish and Spanish counterparts [11].

Southeast and South Asian countries are considered epicentres of antibiotic resistant bacteria [12] with high prevalence of extended spectrum beta- lactamase (ESBL) producing Enterobacteriaceae. In particular, the prevalence of ESBL $E$. coli has been reported to be as high as $50 \%$ in the Vietnamese general population [13], and even higher among infants hospitalized in a neonatal intensive care unit in Taiwan $[14,15]$. In a recent study of healthy infants from Bangladesh, $82 \%$ had stool samples containing third generation cephalosporin-resistant $E$. coli, the majority of which were multidrug-resistant and ESBLproducers [5].

Despite the high prevalence of drug-resistant bacteria in community and hospital settings in Southeast Asia, most literature to date has focused on selected bacteria such as E. coli [16]. This is the first study in the region describing changes in the total antibiotic resistance gene (ARG) profile (also known as the resistome) of healthy infants over time. We aimed to evaluate changes in ARGs over the first year of life in the gut microbiome of infants from the Growing Up in Singapore Towards Healthy Outcomes (GUSTO) study, the largest longitudinal birth cohort study including pregnant women and their infants in Singapore. We also aimed to evaluate the role of demographic and social factors on development of the infant gut resistome.

\section{Methods}

\section{Study population}

The study cohort and methodology of the GUSTO study has been described in detail previously [17]. Briefly, we recruited pregnant mothers aged 18 and above, attending their first trimester antenatal dating scan from the two major maternity units in Singapore who agreed to enrol their offspring for future longitudinal follow-up. Mothers receiving chemotherapy, taking psychotrophic drugs or who had type 1 diabetes mellitus were excluded from the study. Information about subject demographics, family history of allergy, hospitalization, illnesses, social data and lifestyle factors was collected. Structured questionnaires were administered at 3 weeks, 3 months, 6 months and 12 months to collect information on each child's health. Stool samples from enrolled infants were collected at 3 weeks (W3), 3 months (M3), 6 months (M6), and 12 months (M12). We analysed the presence of ARGs in the first year of life, in a subset of 75 infants at risk of eczema from the GUSTO birth cohort. The original aim of the study was to evaluate and compare stool microbiota in infants with eczema vs. those without eczema. Briefly, eczema subjects were selected based on parental report of physician-diagnosed eczema in the first 18 months of life and/or had SCORAD scores at 18 or 36 months [18]. Non-eczema subjects were matched with eczema subjects based on age, mode of delivery (caesarean/vaginal delivered), usage of antibiotics at labour (yes/no), mode of feeding in first 6 months of life (exclusively breastfeeding/partial breastfeeding/total formula feeding) and usage of antibiotics in the first year of life (yes/no). We included 40 non-eczema subjects and 35 eczema subjects. The study flowchart is presented in Fig. 1.

\section{Genomic DNA extraction}

Genomic DNA was extracted from approximately 100$150 \mathrm{mg}$ of stool from each specimen with the ZR Fecal DNA MicroPrep Kit (Zymo Research, USA) according to the manufacturer's instructions.

\section{Metagenomic sequencing \& bioinformatics analysis}

For each stool sample, a sequencing library was first constructed using Illumina's Truseq Nano DNA Library Preparation Kit (Illumina, San Diego, USA). The samples were sheared on a Covaris E220 to $\sim 450 \mathrm{bp}$, following the manufacturer's recommendation, and uniquely tagged with one of Illumina's TruSeq HT DNA barcode combinations to enable sample pooling for sequencing.

The finished libraries were quantitated using Invitrogen's Picogreen assay and the average library size was determined on Bioanalyzer 2100, using a DNA 7500 chip (Agilent). Library concentrations were then normalized to $4 \mathrm{nM}$ and validated by $\mathrm{qPCR}$ on a ViiA-7 real-time 


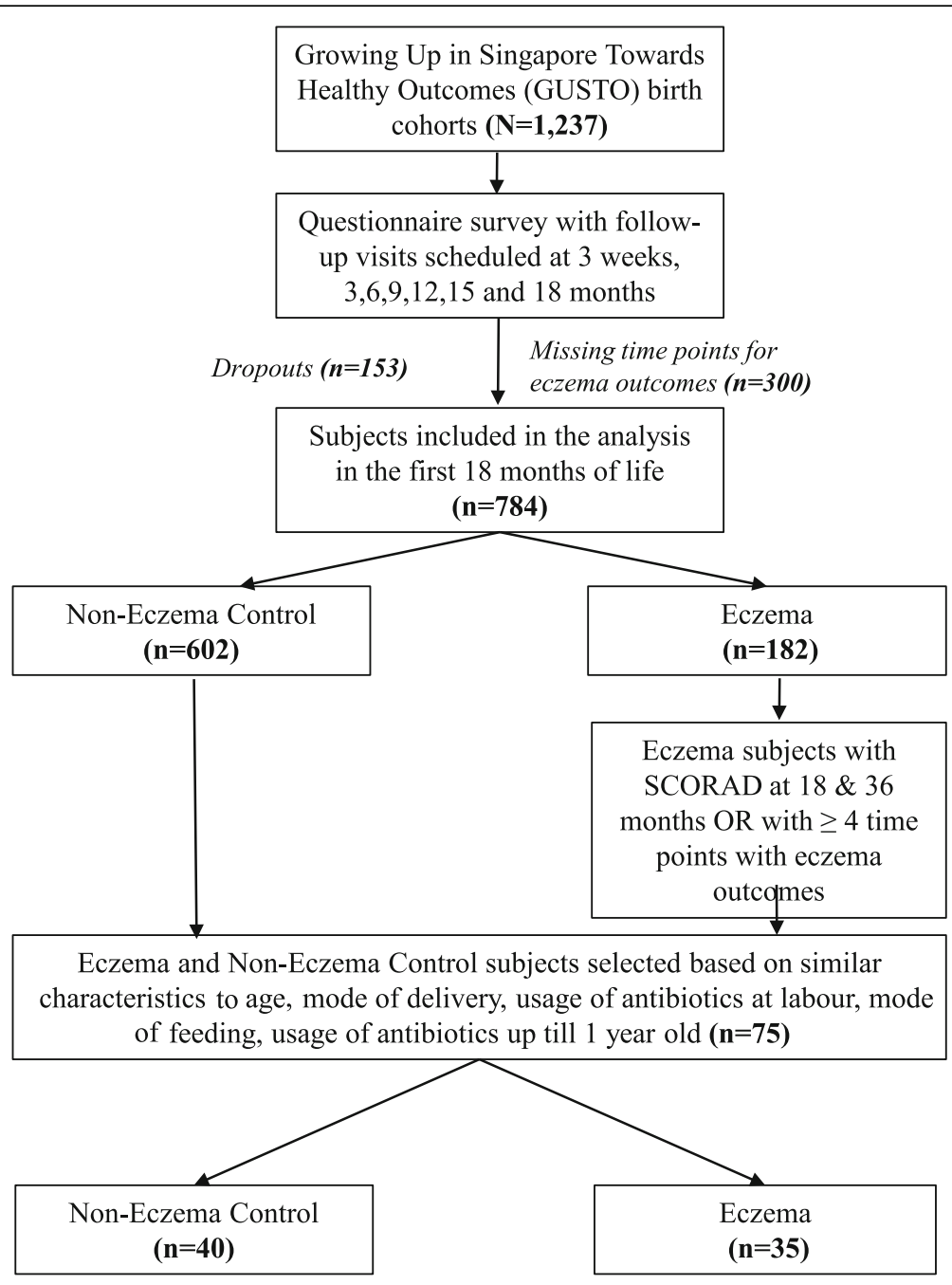

Fig. 1 Flow chart of subject selection

thermocycler (Applied Biosystems), using qPCR primers recommended in Illumina's qPCR protocol, and Illumina's PhiX control library as standard. The libraries were then pooled at equimolar concentrations and sequenced on an Illumina HiSeq2500 sequencer in rapid mode at a read-length of $250 \mathrm{bp}$ paired-end. Approximately $5 \mathrm{~Gb}$ of sequencing data were obtained per sample so as to capture most of the novelty [19].

Genomic DNA sequences obtained from Illumina HiSeq paired-end sequencing were analyzed as follows: (i) Sequence quality check, (ii) Reads to protein alignment, and (iii) Taxonomical classification. To perform a quality check, reads shorter than $30 \mathrm{bp}$ and low-quality sequences $(<\mathrm{Q} 20$ score) were removed. As elaborated by Illumina Inc. (2018), a sequencing quality score of 20 (Q20) represents an average error rate of 1 in 100 bases.

Therefore, removal of any sequence with a higher error rate than 1\% (i.e., <Q20 score) allows for a corresponding call accuracy of at least $99 \%$.
Thereafter, metagenomic reads were obtained by aligning the high-quality reads against human reference genome (hg19), whereby reads that were aligned to hg19 were discarded. The non-human metagenomic reads were then run on ResFinder 2.1. to check for the ARG's conferring resistance to: aminoglycoside, tetracycline, beta-lactam, colistin, fosfomycin, fusidic acid, macrolide, nitroimidazole, oxazolidinone, phenicol, quinolone, rifampicin, sulphonamide, trimethoprim, and glycopeptide antibiotics.

Lastly, a lowest common ancestor (LCA)-based Taxonomical Classification of the aligned sequence reads was carried out on MEGAN6 (MEtaGenome Analyzer 6) using a bitscore cut-off of 100 . A simple algorithm is utilized by MEGAN to assign each read to the LCA of the set of taxa that it hits in the comparison, whereby species-specific sequence reads will be assigned to the species taxon, while widely conserved sequence reads will be assigned to the high-order taxa [20]. 


\section{Statistical analysis}

Univariate analysis using Pearson chi-square test was performed to assess associations between ARGs and demographic, lifestyle and clinical factors.

From the absolute number of normalized reads obtained from the LCA based Taxonomical Classification on MEGAN6, the relative abundance of each bacterial phylum, family, genus and species were calculated. Shannon and Simpson's Diversity Indices were then calculated using relative abundance obtained at the species taxon.

For all statistical analysis, IBM SPSS Statistics (Version 24.0) was used (IBM Corporation, New York, USA), with two-tailed statistical tests and confidence intervals of $95 \%$ (i.e., $p$-value $<0.05$ ). All graphical figures were plotted using Microsoft Excel 2017 (Microsoft Corporation, Washington, USA) and GraphPad Prism Version 7 (GraphPad Software, California USA).

\section{Results}

\section{Study population and clinical characteristics}

The majority of the subjects included in this study were healthy full-term infants $(72 / 75,96.0 \%)$, while 2 were delivered at 36 weeks and 1 at 35 weeks. Out of these 75 infants, $33.3 \%$ were delivered by caesarean delivery (Table 1). The proportion of female and male infants were similar and the majority were of Chinese ethnicity $[42(56 \%)]$. Antibiotics were taken by 35 (46.7\%) mothers during pregnancy and/or labour. Out of the 14 mothers that took antibiotics during pregnancy, 3 took it during the first trimester, 6 during second trimester and 5 during third trimester. A minority of the infants [16 (21.3\%)] were prescribed antibiotics during the first year of life. The subjects included in this study were similar to the larger GUSTO cohort in all characteristics except consumption of antibiotics, illness and hospitalisation (Additional Table 1, Supplementary Data).

\section{Antibiotic resistance gene profile}

There were 188 available stool samples from 75 infants; 28 at week 3, 41 at month 3, 58 at month 6 and 61 at month 12. Eight (10.7\%) subjects had stool samples available at all 4 timepoints. There were 228 unique ARGs detected among all samples. The mean number of ARGs per infant increased with age (23.0 at W3, 25.0 at M3, 25.4 at M6 and 26.0 at M12, $p<0.05$, Fig. 2a) Microbial diversity of the stool samples also increased over time, as measured by the Shannon diversity index (Fig. 2b).

The most common ARGs identified confer resistance to aminoglycoside, beta-lactam, macrolide and tetracycline antibiotics; all infants harboured these ARGs at some point in the first year of life (Additional Figure 1, Supplementary Data). Overall, the most common ARGs were
Table 1 Baseline demographic and clinical characteristics of subjects

\begin{tabular}{|c|c|}
\hline Baseline Demographics & $\begin{array}{l}\text { No. of subjects }(n=75) \\
n(\%)\end{array}$ \\
\hline \multicolumn{2}{|l|}{ Gender } \\
\hline Male & $38(50.7 \%)$ \\
\hline Female & $37(49.3 \%)$ \\
\hline Presence of siblings & $46(61.3 \%)$ \\
\hline \multicolumn{2}{|l|}{ Mode of delivery } \\
\hline Caesarean & $25(33.3 \%)$ \\
\hline Vaginal & $50(66.7 \%)$ \\
\hline \multicolumn{2}{|l|}{ Gestational age } \\
\hline Term ( $\geq 37$ weeks) & $72(96.0 \%)$ \\
\hline Preterm & $3(4.0 \%)$ \\
\hline \multicolumn{2}{|l|}{ Ethnicity } \\
\hline Chinese & $42(56 \%)$ \\
\hline Malay & $21(28 \%)$ \\
\hline Indian & $12(16 \%)$ \\
\hline \multicolumn{2}{|l|}{ Household monthly income } \\
\hline $0-\$ 999$ & $2(2.8 \%)$ \\
\hline$\$ 1000-\$ 1999$ & $9(12.7 \%)$ \\
\hline$\$ 2000-\$ 3999$ & $25(35.2 \%)$ \\
\hline$\$ 4000-\$ 5999$ & $13(18.3 \%)$ \\
\hline$>\$ 6000$ & $22(31 \%)$ \\
\hline Maternal Tertiary Education & $46(61.3 \%)$ \\
\hline Antibiotics during pregnancy and/or labour & $35(46.7 \%)$ \\
\hline \multicolumn{2}{|c|}{ Class of antibiotics used during pregnancy and/ or labour } \\
\hline Beta-lactam & $30(40 \%)$ \\
\hline Macrolide, beta-lactam & $1(1.3 \%)$ \\
\hline Macrolide & $3(4.0 \%)$ \\
\hline Lincosamide & $1(1.3 \%)$ \\
\hline Postnatal antibiotics within first 12 months & $16(21.3 \%)$ \\
\hline Childcare attendance in first year & $7(14 \%)$ \\
\hline Pet ownership at 12 months & $3(6 \%)$ \\
\hline Smoking exposure in first year & $1(1.3 \%)$ \\
\hline Maternal history of atopy & $18(24 \%)$ \\
\hline Paternal history of atopy & $20(26.7 \%)$ \\
\hline Eczema up to 18 months & $35(46.7 \%)$ \\
\hline Rhinitis up to 18 months & $21(28.4 \%)$ \\
\hline Wheeze with nebulizer use up to 18 months & $5(7 \%)$ \\
\hline Allergen sensitization at 18 months & $15(23.1 \%)$ \\
\hline Hospitalisation in the first year & $5(6.7 \%)$ \\
\hline Illness diagnosed in the first year & $38(50.7 \%)$ \\
\hline
\end{tabular}


a)

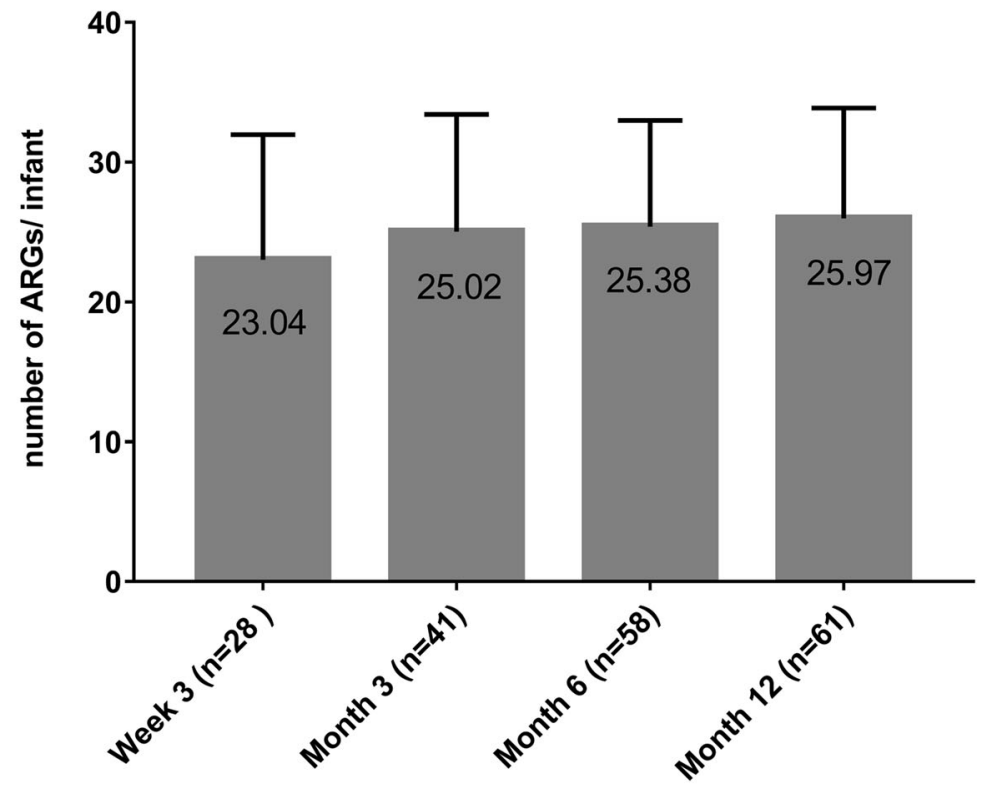

b)

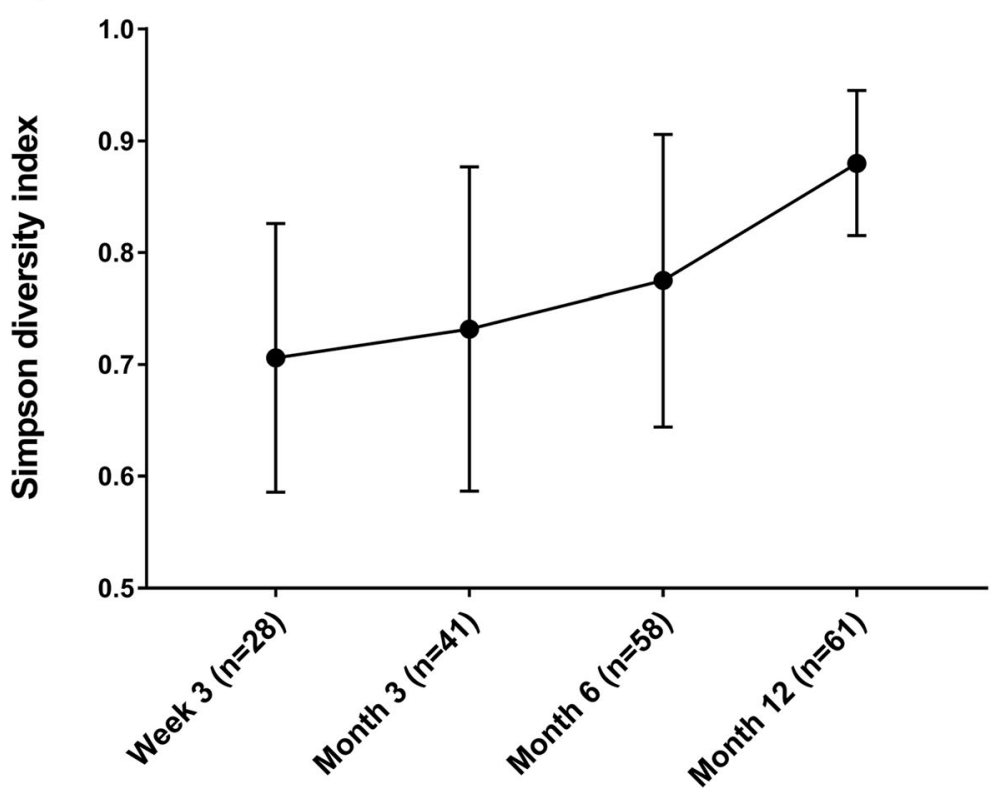

Fig. 2 a Mean number of ARGs per infant over time. b. Microbial diversity of stool samples over time as determined by Simpsons Diversity Index

fosA, blaZ, tet (M) and mef (A), conferring fosfomycin, beta-lactam, tetracycline and macrolide resistance, respectively (Table 2). The most common ARGs detected varied over time. At week 3, the most prevalent ARGs were fosA and blaZ which were present in more than 95\% of infants, while at month 12 the most prevalent ARG was mefA, found in $93.4 \%$ of infants (Table 2).
Association between usage of beta-lactam class of antibiotics during pregnancy and/or labour and acquisition of beta-lactam resistance genes in the offspring

Among the 35 women receiving antibiotics during pregnancy and/or labour, beta-lactam antibiotics were the most commonly given antibiotic class [30/35 (85.7\%)] 
Table 2 Most commonly detected antibiotic resistance genes and associated antibiotic resistance phenotypes by time point

\begin{tabular}{|c|c|c|c|c|c|}
\hline \multirow{2}{*}{\multicolumn{2}{|c|}{$\begin{array}{l}\text { Antibiotic resistance profile and associated } \\
\text { antibiotic resistance genes }\end{array}$}} & \multicolumn{4}{|l|}{ Timepoint } \\
\hline & & \multirow{2}{*}{$\begin{array}{l}\text { W3 } \\
(n=28) \\
\text { No. }(\%) \\
27(96.43 \%)\end{array}$} & \multirow{2}{*}{$\begin{array}{l}\text { M3 } \\
(n=41) \\
\text { No. (\%) } \\
34(82.93 \%)\end{array}$} & \multirow{2}{*}{$\begin{array}{l}\text { M6 } \\
(n=58) \\
\text { No. (\%) } \\
47(81.03 \%)\end{array}$} & \multirow{2}{*}{$\begin{array}{l}\text { M12 } \\
(n=61) \\
\text { No. }(\%) \\
37(60.66 \%)\end{array}$} \\
\hline Fosfomycin & fosA & & & & \\
\hline Beta-lactam & blaz & 27 (96.43\%) & $16(39.02 \%)$ & $12(20.69 \%)$ & $8(13.11 \%)$ \\
\hline Tetracycline & tet(M) & 20 (71.43\%) & $26(63.41 \%)$ & $35(60.34 \%)$ & 38 (62.30\%) \\
\hline Macrolide & $\operatorname{mef}(A)$ & 19 (67.86\%) & 31 (75.61\%) & $46(79.31 \%)$ & 57 (93.44\%) \\
\hline Quinolone & oq $x B$ & 19 (67.86\%) & $24(58.54 \%)$ & $23(39.66 \%)$ & 14 (22.95\%) \\
\hline Quinolone & $\operatorname{msr}(D)$ & $18(64.29 \%)$ & $28(68.29 \%)$ & 47 (81.03\%) & 53 (86.89\%) \\
\hline Macrolide & erm(B) & 17 (60.71\%) & $26(63.41 \%)$ & $48(82.76 \%)$ & $54(88.52 \%)$ \\
\hline Macrolide & Isa(A) & 17 (60.71\%) & 32 (78.05\%) & 29 (50\%) & $5(8.20 \%)$ \\
\hline Tetracycline & tet(W) & $15(53.57 \%)$ & $31(75.61 \%)$ & $51(87.93 \%)$ & 50 (81.97\%) \\
\hline Aminoglycoside & $\operatorname{aac}\left(6^{\prime}\right)-\operatorname{aph}\left(2^{\prime \prime}\right)$ & $15(53.57 \%)$ & $10(24.39 \%)$ & $21(36.21 \%)$ & 34 (55.74\%) \\
\hline
\end{tabular}

The number and percentage of subjects with the antibiotic resistance genes are shown

(Table 1). Details of the antibiotics used are presented in Additional Tables 2 and 3, Supplementary Data.

Given the high usage of the beta-lactam class of antibiotics during pregnancy and/or labour, we next determined if this affected the acquisition of beta-lactam resistance genes in the offspring. All ARGs that conferred beta-lactam resistance were analysed. Offspring of mothers exposed to beta-lactam antibiotics during pregnancy and/or labour had significantly increased detection of the beta-lactam-resistance conferring genes, cepA-44 (9/80, 11.3\%) and cfxA (14/80, 17.5\%), in their stool samples compared to offspring of mothers who did not take these antibiotics $(1 / 101,1.0 \% ; 6 / 101,5.9 \%$ respectively $p<0.01$ ).

There were no significant associations between the use of beta-lactam antibiotics during pregnancy and/or labour and other ARGs conferring beta-lactam resistance in the first year of life. The presence of specific ARGs were not significantly associated with gender, race, delivery mode, postnatal antibiotics use and eczema diagnosis in infancy.

\section{Beta-lactamase type}

We next characterized the type of beta-lactamase genes; the majority were ESBLs [CTX-M beta lactamases (class A), OXA beta-lactamases (class D), SHV betalactamases (class A) and TEM beta-lactamases (class A), Table 3]. The most common ESBL genes was blaTEM$1 \mathrm{~B}$, present in $10(35.7 \%)$ subjects at week 3,22 subjects $(53.7 \%)$ at month 3,34 subjects $(58.6 \%)$ at month 6 , and 29 subjects $(47.5 \%)$ at month 12 . Carbapenemase detection was rare and only CMY (class C) carbapenemases were detected. Among stool samples at each time point, carbapenemase genes were found in $1(3.6 \%)$ at W3, 4 $(9.8 \%)$ at M3, $7(12.0 \%)$ at month 6 and $4(6.6 \%)$ at month 12. Most carbapenemase genes (blaCMY-34, blaCMY-49, blaCMY-83, blaCMY-98) were detected at 1 timepoint only for a given infant, suggesting that they do not persist with time. blaCMY-2 was detected in multiple infants including 1 subject at week 3, 2 at month 3, 4 at month 6 and 2 at month 12. NDM and KPC type carbapenemases were not detected in the infants.

\section{Dynamics of ARGs over time}

We next analysed the most commonly-detected ARGs from early life ( 3 weeks) up to 12 months of age. The analysis was performed among 22 infants who had stool samples at both week 3 (first timepoint) and month 12 (last timepoint). Among these infants, there were 3 ARGs that were present in both samples. These ARGs were $\operatorname{mef}(\mathrm{A})$ conferring macrolide resistance, aadE conferring aminoglycoside resistance, and $\operatorname{msr}(\mathrm{D})$ which conferred macrolide, lincosamide and streptogramin B resistance (Table 4). Among infants who harboured ESBL genes at week 3, we found blaTEM-1b present at month 12 in 6/9 (67\%) subjects and blaOXA-347 in 2/2 (100\%) subjects (Table 5).

An additional subgroup analysis was performed among infants who had stool samples collected at all timepoints. Among the 8 infants who had stool samples analysed at all 4 timepoints, there were 9 ARGs that were present at both week 3 and month 3 samples. These ARGs were lsa(A), $\operatorname{mef}(\mathrm{A}), \operatorname{msr}(\mathrm{D}), \operatorname{sul} 2, \operatorname{tet}(\mathrm{O}), \operatorname{tet}(\mathrm{Q}), \operatorname{tet}(\mathrm{W})$, blaTEM-1B and aadE which conferred macrolide, macrolide, sulphonamide, tetracycline, beta-lactam, aminoglycoside, macrolide, lincosamide and streptogramin $\mathrm{B}$ resistance respectively. There were 4 ARGs that were present at all timepoints from week 3 to month 6 and they were tet $(\mathrm{O})$, tet $(\mathrm{Q})$, tet $(\mathrm{W})$, blaTEM-1B, which confer tetracycline and beta-lactam resistance. There were 
Table 3 Detected beta-lactamase genes

\begin{tabular}{|c|c|c|c|c|c|}
\hline \multicolumn{2}{|l|}{ Beta-lactam resistance genes } & $\begin{array}{l}\text { W3 } \\
n=28\end{array}$ & $\begin{array}{l}\text { M3 } \\
n=41\end{array}$ & $\begin{array}{l}M 6 \\
n=58\end{array}$ & $\begin{array}{l}M 12 \\
n=61\end{array}$ \\
\hline \multicolumn{6}{|l|}{ Carbapenemases } \\
\hline \multirow[t]{6}{*}{ CMY (Class C) } & blaCMY-2 & 1 & 2 & 4 & 2 \\
\hline & blaCMY-34 & 0 & 0 & 0 & 1 \\
\hline & blaCMY-49 & 0 & 1 & 0 & 0 \\
\hline & blaCMY-76 & 0 & 0 & 2 & 1 \\
\hline & blaCMY-83 & 0 & 0 & 1 & 0 \\
\hline & blaCMY-98 & 0 & 1 & 0 & 0 \\
\hline \multicolumn{6}{|l|}{ Extended spectrum beta-lactamases } \\
\hline \multirow[t]{6}{*}{ CTX-M beta lactamases (Class A) } & blaCTX-M-14 & 0 & 1 & 1 & 0 \\
\hline & blaCTX-M-15 & 0 & 1 & 0 & 1 \\
\hline & blaCTX-M-27 & 0 & 1 & 1 & 0 \\
\hline & blaCTX-M-40 & 0 & 0 & 1 & 1 \\
\hline & blaCTX-M-78 & 0 & 0 & 0 & 1 \\
\hline & blaCTX-M-8 & 0 & 0 & 2 & 1 \\
\hline \multirow[t]{7}{*}{ OXA beta-lactamases (class D) } & blaOXA-1 & 0 & 0 & 1 & 2 \\
\hline & blaOXA-10 & 0 & 1 & 0 & 0 \\
\hline & blaOXA-116 & 1 & 0 & 0 & 0 \\
\hline & blaOXA-120 & 0 & 0 & 1 & 0 \\
\hline & blaOXA-184 & 0 & 0 & 0 & 1 \\
\hline & blaOXA-347 & 2 & 9 & 11 & 14 \\
\hline & blaOXA-85 & 1 & 0 & 1 & 0 \\
\hline \multirow[t]{13}{*}{ SHV beta-lactamases (Class A) } & blaSHV-1 & 9 & 9 & 7 & 3 \\
\hline & blaSHV-108 & 0 & 0 & 1 & 0 \\
\hline & blaSHV-11 & 5 & 8 & 7 & 4 \\
\hline & blaSHV-142 & 1 & 0 & 0 & 0 \\
\hline & blaSHV-26 & 1 & 0 & 0 & 0 \\
\hline & blaSHV-27 & 0 & 0 & 0 & 4 \\
\hline & blaSHV-28 & 1 & 0 & 0 & 0 \\
\hline & blaSHV-38 & 0 & 0 & 1 & 0 \\
\hline & blaSHV-63 & 0 & 0 & 0 & 1 \\
\hline & blaSHV-75 & 0 & 0 & 1 & 0 \\
\hline & blaSHV-83 & 1 & 2 & 0 & 0 \\
\hline & blaSHV-85 & 0 & 0 & 0 & 1 \\
\hline & blaSHV-93 & 0 & 1 & 1 & 0 \\
\hline \multirow[t]{4}{*}{ TEM beta-lactamases (Class A) } & blaTEM-176 & 1 & 1 & 1 & 2 \\
\hline & blaTEM-1B & 10 & 22 & 34 & 29 \\
\hline & blaTEM-1C & 0 & 0 & 0 & 3 \\
\hline & blaTEM-1D & 0 & 0 & 0 & 1 \\
\hline
\end{tabular}

The number of subjects with beta lactam resistance genes detected in their stool samples at each time point are shown

only 2 ARGs that were present at all timepoints from week 3 to month 12: tet $(\mathrm{Q})$ from Bacteroides and tet $(\mathrm{W})$ from Bifidobacterium, both conferring tetracycline resistance (Table 4).
Beta-lactam resistant $E$. coli and $K$. pneumoniae

Beta-lactam resistant E. coli, which is endemic in Singapore, was detected in $4(5.3 \%)$ subjects at different timepoints, including, 1 subject at week 3,2 at 
Table 4 Presence of antibiotic resistance genes (ARGs) at time points during the first year of life

\begin{tabular}{|c|c|c|c|c|c|c|c|c|c|c|c|}
\hline \multirow[b]{2}{*}{ Antibiotic resistance genotype } & \multirow[b]{2}{*}{ ARG } & \multicolumn{2}{|c|}{$\begin{array}{l}\text { Number of } \\
\text { subjects\# }\end{array}$} & \multirow{2}{*}{$\begin{array}{l}\% \text { of subjects with ARG present } \\
\text { at time points } \\
\text { W3 and M12 }\end{array}$} & \multicolumn{4}{|c|}{ Number of subjects* } & \multicolumn{3}{|c|}{$\begin{array}{l}\% \text { of subjects with ARG } \\
\text { present at time points }\end{array}$} \\
\hline & & W3 & $\begin{array}{l}\text { W3 and } \\
\text { M12 }\end{array}$ & & W3 & $\begin{array}{l}\text { W3 to } \\
\text { M3 }\end{array}$ & $\begin{array}{l}\text { W3 to } \\
\text { M6 }\end{array}$ & $\begin{array}{l}\text { W3 to } \\
\text { M12 }\end{array}$ & $\begin{array}{l}\text { W3 to } \\
\text { M3 }\end{array}$ & $\begin{array}{l}\text { W3 to } \\
\text { M6 }\end{array}$ & $\begin{array}{l}\text { W3 to } \\
\text { M12 }\end{array}$ \\
\hline Fosfomycin & fos $\mathrm{A}$ & 21 & 11 & $52 \%$ & 8 & 7 & 6 & 4 & $88 \%$ & $75 \%$ & $50 \%$ \\
\hline Beta-lactam & blaZ & 21 & 2 & $10 \%$ & 7 & 1 & 1 & 0 & $14 \%$ & $14 \%$ & $0 \%$ \\
\hline Aminoglycoside & $\begin{array}{l}\operatorname{aac}\left(6^{\prime}\right)- \\
\operatorname{aph}\left(2^{\prime \prime}\right)\end{array}$ & 11 & 5 & $45 \%$ & 5 & 1 & 0 & 0 & $20 \%$ & $0 \%$ & $0 \%$ \\
\hline Macrolide & $\operatorname{Isa}(A)$ & 13 & 2 & $15 \%$ & 5 & 5 & 3 & 1 & $100 \%$ & $60 \%$ & $20 \%$ \\
\hline Quinolone & oqxB & 14 & 4 & $29 \%$ & 5 & 4 & 1 & 1 & $80 \%$ & $20 \%$ & $20 \%$ \\
\hline Macrolide & erm(B) & 13 & 11 & $85 \%$ & 4 & 2 & 2 & 2 & $50 \%$ & $50 \%$ & $50 \%$ \\
\hline Macrolide & $\operatorname{erm}(X)$ & 6 & 5 & $83 \%$ & 4 & 3 & 2 & 1 & $75 \%$ & $50 \%$ & $25 \%$ \\
\hline Macrolide & $\operatorname{mef}(A)$ & 15 & 15 & $100 \%$ & 4 & 4 & 3 & 3 & $100 \%$ & $75 \%$ & $75 \%$ \\
\hline $\begin{array}{l}\text { Macrolide, Lincosamide and } \\
\text { Streptogramin B }\end{array}$ & msr(D) & 14 & 14 & $100 \%$ & 4 & 4 & 2 & 2 & $100 \%$ & $50 \%$ & $50 \%$ \\
\hline Tetracycline & tet(M) & 15 & 11 & $73 \%$ & 4 & 2 & 1 & 1 & $50 \%$ & $25 \%$ & $25 \%$ \\
\hline Aminoglycoside & $\operatorname{aph}\left(3^{\prime}\right)-|| \mid$ & 7 & 5 & $71 \%$ & 3 & 2 & 1 & 1 & $67 \%$ & $33 \%$ & $33 \%$ \\
\hline Sulphonamide & sul2 & 8 & 4 & $50 \%$ & 3 & 3 & 2 & 2 & $100 \%$ & $67 \%$ & $67 \%$ \\
\hline Tetracycline & tet(O) & 6 & 4 & $67 \%$ & 3 & 3 & 3 & 2 & $100 \%$ & $100 \%$ & $67 \%$ \\
\hline Tetracycline & $\operatorname{tet}(\mathrm{Q})$ & 5 & 4 & $80 \%$ & 3 & 3 & 3 & 3 & $100 \%$ & $100 \%$ & $100 \%$ \\
\hline Tetracycline & $\operatorname{tet}(\mathrm{W})$ & 11 & 10 & $91 \%$ & 3 & 3 & 3 & 3 & $100 \%$ & $100 \%$ & $100 \%$ \\
\hline Beta-lactam & $\begin{array}{l}\text { blaTEM- } \\
1 \mathrm{~b}\end{array}$ & 9 & 6 & $67 \%$ & 2 & 2 & 2 & 1 & $100 \%$ & $100 \%$ & $50 \%$ \\
\hline Aminoglycoside & aadE & 1 & 1 & $100 \%$ & 1 & 1 & 0 & 0 & $100 \%$ & $0 \%$ & $0 \%$ \\
\hline
\end{tabular}

\# subjects with stool samples at both week 3 and month $12(N=22) .{ }^{*}$ subjects with stool samples at all timepoints $(N=8)$

month 3 and 2 at month 6 respectively. No betalactam resistant $E$. coli was detected at month 12 . There were 3 ESBL genes detected in E. coli, namely blaCMY-2, blaCTX-M15 and blaTEM-1b (Table 6). In contrast, beta-lactam resistant $K$. pneumoniae was more common and was detected in 32 (42.7\%) subjects at any time point. The prevalence of beta-lactam resistant $K$. pneumoniae declined over time; it was found in 13 subjects at week 3, 10 at month 3, 11 at month 6,8 at month 12 . Among these K. pneumoniae, there were 9 ESBLs detected, all of which were SHV beta-lactamases (class A) (Table 6).

\section{Discussion}

We report the first longitudinal cohort study in Southeast Asia to evaluate the diversity and dynamics of the infant gut resistome over the first year of life. We

Table 5 Presence of extended spectrum beta-lactamase (ESBL) resistance genes in the first year of life

\begin{tabular}{|c|c|c|c|c|c|c|c|c|c|c|}
\hline \multirow[b]{2}{*}{ Extended Spectrum Beta-lactamases } & \multicolumn{2}{|c|}{$\begin{array}{l}\text { Number of } \\
\text { subjects\# }\end{array}$} & \multirow{2}{*}{$\begin{array}{l}\text { Presence } \\
\text { W3 and M12 }\end{array}$} & \multicolumn{4}{|c|}{ Number of subjects* } & \multicolumn{3}{|l|}{ Presence } \\
\hline & W3 & $\mathrm{W} 3$ and $\mathrm{M} 12$ & & W3 & W3 to M3 & W3 to M6 & W3 to $\mathrm{M} 12$ & W3 to M3 & W3 to M6 & W3 to $\mathrm{M} 12$ \\
\hline blaTEM-1b & 9 & 6 & $67 \%$ & 2 & 2 & 2 & 1 & $100 \%$ & $100 \%$ & $50 \%$ \\
\hline blaSHV-1 & 8 & 1 & $13 \%$ & 2 & 0 & 0 & 0 & $0 \%$ & $0 \%$ & $0 \%$ \\
\hline blaSHV-11 & 3 & 0 & $33 \%$ & 1 & 1 & 0 & 0 & $100 \%$ & $0 \%$ & $0 \%$ \\
\hline blaSHV-142 & 1 & 0 & $0 \%$ & 1 & 0 & 0 & 0 & $0 \%$ & $0 \%$ & $0 \%$ \\
\hline blaSHV-83 & 1 & 0 & $0 \%$ & 1 & 0 & 0 & 0 & $0 \%$ & $0 \%$ & $0 \%$ \\
\hline blaOXA-116 & 1 & 0 & $0 \%$ & 1 & 0 & 0 & 0 & $0 \%$ & $0 \%$ & $0 \%$ \\
\hline blaOXA-347 & 2 & 2 & $100 \%$ & 1 & 1 & 1 & 1 & $100 \%$ & $100 \%$ & $100 \%$ \\
\hline
\end{tabular}

\# subjects with stool samples at both week 3 and month $12(N=22){ }^{*}$ subjects with stool samples at all timepoints $(N=8)$ 
Table 6 Extended spectrum beta-lactamase genes found in E. coli and Klebsiella pneumoniae

\begin{tabular}{llllll}
\hline Extended-Spectrum Beta-lactam resistance genes & Species & W3 & M3 & M6 & M12 \\
\hline CMY (Class C) & blaCMY-2 & Escherichia coli & 1 & 1 & 1 \\
CTX-M beta lactamases (Class A) & blaCTX-M15 & Escherichia coli & 0 & 1 & 0 \\
SHV beta-lactamases (Class A) & blaSHV-1 & Klebsiella pneumoniae & 6 & 7 & 4 \\
& blaSHV-108 & Klebsiella pneumoniae & 0 & 0 & 1 \\
& blaSHV-11 & Klebsiella pneumoniae & 5 & 2 & 4 \\
& blaSHV-142 & Klebsiella pneumoniae & 1 & 0 & 0 \\
& blaSHV-26 & Klebsiella pneumoniae & 1 & 0 & 0 \\
& blaSHV-27 & Klebsiella pneumoniae & 0 & 0 & 0 \\
& blaSHV-38 & Klebsiella pneumoniae & 0 & 0 & 1 \\
& blaSHV-85 & Klebsiella pneumoniae & 0 & 0 & 0 \\
TEM beta-lactamases (Class A) & blaSHV-93 & Klebsiella pneumoniae & 0 & 1 & 0 \\
\hline
\end{tabular}

observed that in an area with high endemic bacterial drug resistance, the infant gut harbours a wide diversity of ARGs from early in infancy [4, 6-9]. We also observed significant differences among the ARGs prevalent in early infancy compared to those prevalent at 1 year of life. Generally, the number of ARGs per infant stool specimen increased over time during the first year of life. This increasing prevalence may be due to environmental acquisition of some genes or the emergence of resistance among normal flora. A minority of ARGs persisted through the first year of life, demonstrating a highly dynamic infant resistome [21, 22].

Our findings are supported by studies from other parts of the world. Gosalbes and colleagues found a high prevalence of resistance to beta-lactam antibiotics and tetracycline in both meconium and stool samples from 1-weekold infants, suggesting the acquisition of the infant gut resistance reservoir even before birth [22]. A functional screen for ARGs in the gut microbiota of 22 healthy 6month old infants from Ireland, who had not received any prior antibiotics, demonstrated the presence of a variety of genes encoding resistance to aminoglycosides and betalactams [23]. Nogacka and colleagues also found a higher occurrence of some beta-lactamase encoding genes in vaginally-delivered term Spanish infants whose mothers received intra-partum antimicrobial prophylaxis, compared to those whose mothers did not receive intrapartum antibiotics [24]. Wintersdorff et al. also noted increasing prevalence of ARGs over time in infants, in Germany [25].

The prevalence of ARGs encoding resistance to antibiotics that are rarely used in humans, such as chloramphenicol, may imply selection pressure from the environment, or food and agriculture, possibly via maternal diet. If so, this is likely to vary across different regions throughout the world. Early in infancy, certain
ARGs may be easily acquired from breast milk or even environmental contaminants of formula milk [26, 27]. Later on, as infants are weaned and begin eating table foods, ARGs may be acquired from ingesting meat and vegetables. It is known that antibiotics are often used in livestock for growth promotion and disease prevention, particularly in settings of intensive animal production [28]. This has been known to be implicated in the development of antimicrobial-resistant bacteria that can be spread to humans. The tendency to acquire or lose ARGs may be additionally affected by a complex interaction of epigenetic factors, but the large diversity of ARGs may make it challenging to find significant associations between specific ARGs and the various clinical variables studied.

We observed that maternal beta-lactam exposure during pregnancy or delivery was associated with the detection of beta-lactam resistance genes among infants. This is consistent with the work by Nogacka et al. [24], who found that intra-partum antibiotics affect the composition of the infant gut microbiome.

Surprisingly, we did not observe any association between the mode of delivery and acquisition of ARGs in the offspring. This finding differs from a previous study which showed that tetracycline resistance genes such as tet $(M)$, tet $(O)$, tet $(W)$, and tet $(Q)$ were identified in maternal vaginal flora, and that their transmission to offspring was dependent on mode of delivery; $50 \%$ of the infants delivered vaginally harboured tetracycline resistance genes tet $(\mathrm{M})$ and tet $(\mathrm{O})$ found in mothers, while 16 and $13 \%$ of infants delivered by $\mathrm{C}$-section had tet $(\mathrm{O})$ and tet(W) respectively [9].

The prevalence of ARGs in the gut resistome did not always correlate with common multidrug resistance phenotypes in the community. For example, mecA, which confers resistance to all beta-lactam antibiotics and is 
found in most methicillin-resistant Staphylococcus aureus (MRSA) strains, was not prevalent in the infant resistome despite its high incidence in adults in the hospital and community [29]. Similarly, beta-lactamresistant E. coli, which is endemic in Singapore, was only found in $5 \%$ of the infants. In contrast, beta-lactamresistant $K$. pneumoniae, which is also common in the community, was found in $42 \%$ of the infants.

In our study, we also found several ESBL genes in infants' stools in the first year of life, independent of the selection pressure of antibiotic exposure. This highlights the endemicity of these strains in Singapore, and the global problem of transmission of multi-drug antibiotic resistant bacteria. Surprisingly few infants harboured drugresistant E. coli, despite widespread dissemination of drug-resistant E. coli sequence types in Singapore [3033]. In contrast, drug-resistant $K$. pneumoniae was detected in a number of infants from the GUSTO cohort. K. pneumoniae is known to acquire ARGs via de novo mutations, plasmid dissemination and transfer of ARGs. This underscores the potential for the infant gut resistome to facilitate the rise of extremely drug resistant (XDR) strains carrying 'super resistomes' [34].

The infant gut microbiome in dysbiosis has been implicated in diseases such as necrotizing enterocolitis and allergic disease. However, the significance of the infant gut resistome specifically and its role in human disease is still unclear. It is not known what constitutes a "healthy" resistome and the implications of ARG diversity in the infant gut. It may be a reflection of our antimicrobial use not just in pregnancy but also in the food industry. It may have longer lasting implications on the nature and severity of bacterial infections acquired later on in life. Potentially, the infant gut resistome can influence the incidence of multi-drug resistant (MDR) infections, by harbouring ARGs in commensals that transfer to pathogenic bacteria [10]. Better understanding of the naïve infant gut resistome may help to build the evidence base for antimicrobial stewardship.

The strengths of this study are the longitudinal collection of infants' stool specimens and follow up of the infants by structured questionnaires in early life. In addition, the large sample size and higher endemic resistance rates in Singapore are also strengths compared to earlier studies. However, a limitation of this study is that maternal stool specimens and infant stool specimens at birth were not available. It is hence not possible to identify which ARGs were likely to have been vertically transmitted. In addition, not all infants had stool samples available at all time points, thereby limiting analyses of ARG persistence over time. The large diversity of ARGs may also make it challenging to find clinically significant associations between specific ARGs and demographic and clinical variables. Our studied population also overrepresents subjects with eczema, limiting its generalizability as they may carry a higher risk of ARG carriage than the baseline population.

\section{Conclusion}

In conclusion, this study describes the diversity and dynamic nature of the infant gut resistome in a region with high endemic resistance rates, and the paucity of known variables that affect the diversity of the gut resistome. Future work should focus on the mode of acquisition, transmission, and persistence of these ARGs. Metagenomic comparisons between microbiota in infants and their adult household contacts would also help elucidate factors influencing the establishment of the infant gut resistome.

\section{Supplementary information}

Supplementary information accompanies this paper at https://doi.org/10. 1186/s12879-020-05000-y.

Additional file 1: Table S1. Comparison of demographic variables between subjects included and excluded from study. Figure S1. Antibiotic resistance genotypes over time. The percentage of subjects with the antibiotic resistance genotype are presented over time. Table S2. Maternal antibiotic exposures during pregnancy. Table S3. Maternal antibiotic exposures during labour.

\section{Abbreviations}

ARGs: Antibiotic resistance genes; ESBL: Extended spectrum betalactamases.; GUSTO: Growing Up in Singapore Towards Healthy Outcomes

\section{Acknowledgements}

We thank the GUSTO study group and all clinical and home-visit staff involved. The voluntary participation of all subjects is greatly appreciated. The GUSTO study group includes Airu Chia, Allan Sheppard, Amutha Chinnadurai, Anna Magdalena Fogel, Anne Eng Neo Goh, Anne Hin Yee Chu, Anne Rifkin-Graboi, Anqi Qiu, Arijit Biswas, Bee Wah Lee, Birit Froukje Philipp Broekman, Bobby Kyungbeom Cheon, Boon Long Quah, Candida Vaz, Chai Kiat Chng, Cheryl Shufen Ngo, Choon Looi Bong, Christiani Jeyakumar Henry, Ciaran Gerard Forde, Claudia Chi, Daniel Yam Thiam Goh, Dawn Xin Ping Koh, Desiree Y. Phua, Doris Ngiuk Lan Loh, E Shyong Tai, Elaine Kwang Hsia Tham, Elaine Phaik Ling Quah, Evelyn Chung Ning Law, Evelyn Xiu Ling Loo, Fabian Kok Peng Yap, Faidon Magkos, Falk Mueller-Riemenschneider, George Seow Heong Yeo, Hannah Ee Juen Yong, Helen Yu Chen, Heng Hao Tan, Hong Pan, Hugo P S van Bever, Hui Min Tan, Iliana Magiati, Inez Bik Yun Wong, Ivy Yee-Man Lau, Izzuddin Bin Mohd Aris, Jeannie Tay, Jeevesh Kapur, Jenny L. Richmond, Jerry Kok Yen Chan, Jia Xu, Joanna Dawn Holbrook, Joanne Su-Yin Yoong, Joao Nuno Andrade Requicha Ferreira, Jonathan Tze Liang Choo, Jonathan Y. Bernard, Jonathan Yinhao Huang, Joshua J. Gooley, Jun Shi Lai, Karen Mei Ling Tan, Keith M. Godfrey, Kenneth Yung Chiang Kwek, Keri McCrickerd, Kothandaraman Narasimhan, Krishnamoorthy Naiduvaje, Kuan Jin Lee, Leher Singh, Li Chen, Lieng Hsi Ling, Lin Lin Su, Ling-Wei Chen, Lourdes Mary Daniel, Lynette Pei-Chi Shek, Marielle V. Fortier, Mark Hanson, Mary Foong-Fong Chong, Mary Rauff, Mei Chien Chua, Melvin KheeShing Leow, Michael J. Meaney, Michelle Zhi Ling Kee, Min Gong, Mya Thway Tint, Navin Michael, Neerja Karnani, Ngee Lek, Oon Hoe Teoh, P. C. Wong, Paulin Tay Straughan, Pratibha Keshav Agarwal, Priti Mishra, Queenie Ling Jun Li, Rob Martinus van Dam, Salome A. Rebello, Sambasivam Sendhil Velan, Seang Mei Saw, See Ling Loy, Seng Bin Ang, Shang Chee Chong, Sharon Ng, Shiao-Yng Chan, Shirong Cai, Shu-E Soh, Sok Bee Lim, Stella Tsotsi, Stephen Chin-Ying Hsu, Sue-Anne Ee Shiow Toh, Suresh Anand Sadananthan, Swee Chye Quek, Varsha Gupta, Victor Samuel Rajadurai, Walter Stunkel, Wayne Cutfield, Wee Meng Han, Wei Wei Pang, Wen Lun Yuan, Yanan Zhu, Yin Bun Cheung, Yiong Huak Chan, Yung Seng Lee. 


\section{Authors' contributions}

EXL and $A Z$ analysed, interpreted the data and wrote the manuscript. GCY analysed and interpreted the data. RWP, DIDM, YQK, SK and SCS analysed the data. YSC, KHT, PDG, FY, JGE and LPS were involved in the design of the study. ET revised the manuscript. RB and BWL supervised the research and revised the manuscript. All authors reviewed the manuscript and approved the submitted version.

\section{Funding}

The study was supported by the National Medical Research Council Clinician Scientists Individual Research Grant (R-178-000-222-511) provided by the Ministry of Health (Singapore). This research is also supported by the Singapore National Research Foundation under its Translational and Clinical Research (TCR) Flagship Programme and administered by the Singapore Ministry of Health's National Medical Research Council (NMRC), SingaporeNMRC/TCR/004-NUS/2008; NMRC/TCR/012-NUHS/2014. Additional funding is provided by the Singapore Institute for Clinical Sciences, Agency for Science Technology and Research (A*STAR), Singapore. The funders are not involved in the design and conduct of the study, data analysis and preparation of manuscript.

\section{Availability of data and materials}

The datasets used and/or analysed during the current study are available from the corresponding author on reasonable request.

\section{Ethics approval and consent to participate}

Ethics approval was obtained from the Domain Specific Review Board of Singapore National Healthcare Group and the Centralised Institutional Review Board of SingHealth. Conduct of the study was based on the guidelines in the Declaration of Helsinki. Written informed consent was obtained from all participants.

\section{Consent for publication}

Not applicable.

\section{Competing interests}

YSC has received reimbursement for speaking at conferences sponsored by Abbott Nutrition, Nestle, and Danone. LPS has received reimbursement for speaking at conferences sponsored by Danone and Nestle and consulting for Mead Johnson and Nestle.

YSC is part of an academic consortium that has received research funding from Abbot Nutrition, Nestle and Danone. LPS has received research funding from Danone.

\section{Author details}

${ }^{1}$ Singapore Institute for Clinical Sciences (SICS), Agency for Science, Technology and Research (A*STAR), Singapore, Singapore. ${ }^{2}$ Department of Paediatrics, Yong Loo Lin School of Medicine, National University of Singapore, Singapore, Singapore. ${ }^{3}$ Khoo Teck Puat-National University Children's Medical Institute, National University Hospital, National University Health System, Singapore, Singapore. ${ }^{4}$ Singapore Centre For Environmental Life Sciences Engineering (SCELSE), Nanyang Technological University, Singapore, Singapore. ${ }^{5}$ Department of Obstetrics \& Gynaecology, Yong Loo Lin School of Medicine, National University of Singapore and National University Health System, Singapore, Singapore. ${ }^{6}$ Department of Maternal Fetal Medicine, KK Women's and Children's Hospital (KKH), Singapore, Singapore. 'Liggins Institute, University of Auckland, Auckland, New Zealand. ${ }^{8}$ Department of Endocrinology KK Women's and Children's Hospital (KKH), Singapore, Singapore. ${ }^{9}$ Vanderbilt University Medical Center, Nashville, TN, USA.

\section{Received: 26 November 2019 Accepted: 29 March 2020} Published online: 28 April 2020

\section{References}

1. Warnes SL, Highmore CJ, Keevil CW. Horizontal transfer of antibiotic resistance genes on abiotic touch surfaces: implications for public health. MBio. 2012;3(6):e00489-12.

2. Bush K, Fisher JF. Epidemiological expansion, structural studies, and clinical challenges of new $\beta$-lactamases from gram-negative bacteria. Ann Rev Microbiol. 2011;65:455-78.
3. Bonomo RA. New Delhi metallo- $\beta$-lactamase and multidrug resistance: a global SOS? Clin Infect Dis. 2011;52(4):485-7.

4. Gueimonde M, Salminen S, Isolauri E. Presence of specific antibiotic (tet) resistance genes in infant faecal microbiota. FEMS Immunol Med Microbiol. 2006;48(1):21-5.

5. Islam MA, Amin MB, Roy S, Asaduzzaman M, Islam MR, Navab-Daneshmand T, et al. Fecal colonization with multidrug-resistant $E$. coli among healthy infants in rural Bangladesh. Front Microbiol. 2019;10:640.

6. Mitsou E, Kirtzalidou E, Pramateftaki P, Kyriacou A. Antibiotic resistance in faecal microbiota of Greek healthy infants. Benefic Microbes. 2010;1(3):297306.

7. De Vries LE, Vallès Y, Agersø Y, Vaishampayan PA, García-Montaner A, Kuehl $J V$, et al. The gut as reservoir of antibiotic resistance: microbial diversity of tetracycline resistance in mother and infant. PLoS One. 2011;6(6):e21644.

8. Zhang L, Kinkelaar D, Huang Y, Li Y, Li X, Wang HH. Acquired antibiotic resistance: are we born with it? Appl Environ Microbiol. 2011;AEM:05087-11.

9. Alicea-Serrano AM, Contreras M, Magris M, Hidalgo G, Dominguez-Bello MG. Tetracycline resistance genes acquired at birth. Arch Microbiol. 2013;195(6): 447-51.

10. Baron SA, Diene SM, Rolain J-M. Human microbiomes and antibiotic resistance. Hum Microbiome J. 2018;10:43-52.

11. Hu Y, Yang X, Qin J, Lu N, Cheng G, Wu N, et al. Metagenome-wide analysis of antibiotic resistance genes in a large cohort of human gut microbiota. Nat Commun. 2013;4:2151.

12. Jean SS, Hsueh PR. High burden of antimicrobial resistance in Asia. Int J Antimicrob Agents. 2011;37(4):291-5.

13. Bui TM, Hirai I, Ueda S, Bui TK, Hamamoto K, Toyosato T, et al. Carriage of Escherichia coli producing CTX-M-type extended-spectrum beta-lactamase in healthy Vietnamese individuals. Antimicrob Agents Chemother. 2015; 59(10):6611-4.

14. Tsai M-H, Chu S-M, Hsu J-F, Lien R, Huang H-R, Chiang M-C, et al. Risk factors and outcomes for multidrug-resistant gram-negative bacteremia in the NICU. Pediatrics. 2014;133(2):e322-9.

15. Folgori L, Bielicki J, Heath PT, Sharland M. Antimicrobial-resistant gramnegative infections in neonates: burden of disease and challenges in treatment. Curr Opin Infect Dis. 2017;30(3):281-8.

16. Hetzer B, Orth-Holler D, Wurzner R, Kreidl P, Lackner M, Muller T, et al. Enhanced acquisition of antibiotic-resistant intestinal $E$. coli during the first year of life assessed in a prospective cohort study. Antimicrob Resist Infect Contr. 2019:8:79.

17. Soh SE, Lee SSM, Hoon SW, Tan MY, Goh A, Lee BW, et al. The methodology of the GUSTO cohort study: a novel approach in studying pediatric allergy. Asia Pacific Allergy. 2012;2(2):144-8.

18. European Task Force on Atopic Dermatitis. Severity scoring of atopic dermatitis: the SCORAD index. Consensus report of the European task force on atopic dermatitis. Dermatology. 1993;186(1):23-31.

19. Qin J, Li R, Raes J, et al. A human gut microbial gene catalogue established by metagenomic sequencing. Nature. 2010;464:59-65.

20. Huson DH, et al. Integrative analysis of environmental sequences using MEGAN4. Genome Res. 2011;21(9):1552-60.

21. Bäckhed F, Roswall J, Peng Y, Feng Q, Jia H, Kovatcheva-Datchary P, et al. Dynamics and stabilization of the human gut microbiome during the first year of life. Cell Host Microbe. 2015;17(5):690-703.

22. Gosalbes $M$, Vallès $Y$, Jiménez-Hernández $N$, Balle $C$, Riva P, Miravet-Verde $S$, et al. High frequencies of antibiotic resistance genes in infants' meconium and early fecal samples. J Dev Orig Health Dis. 2016;7(1):35-44.

23. Fouhy F, Ogilvie LA, Jones BV, Ross RP, Ryan AC, Dempsey EM, et al. Identification of aminoglycoside and $\beta$-lactam resistance genes from within an infant gut functional metagenomic library. PLoS One. 2014;9(9):e108016.

24. Nogacka A, Salazar N, Suárez M, Milani C, Arboleya S, Solís G, et al. Impact of intrapartum antimicrobial prophylaxis upon the intestinal microbiota and the prevalence of antibiotic resistance genes in vaginally delivered full-term neonates. Microbiome. 2017;5(1):93.

25. Wintersdorff CJv, Wolffs PF, Savelkoul PH, Nijsen RR, Lau S, Gerhold K, et al. The gut resistome is highly dynamic during the first months of life. Future Microbiol. 2016;11(4):501-10.

26. Pärnänen $K$, Karkman A, Hultman J, Lyra C, Bengtsson-Palme J, Larsson DJ, et al. Maternal gut and breast milk microbiota affect infant gut antibiotic resistome and mobile genetic elements. Nat Commun. 2018;9(1):3891.

27. Yassour $M$, Vatanen $T$, Siljander $H$, Hämäläinen $A-M$, Härkönen $T$, Ryhänen SJ, et al. Natural history of the infant gut microbiome and impact of 
antibiotic treatment on bacterial strain diversity and stability. Sci Transl Med. 2016;8(343):343ra81.

28. World Health Organization. Antimicrobial resistance in the food chain. 2017. https://www.who.int/foodsafety/areas_work/antimicrobial-resistance/ amrfoodchain/en/. Accessed 23 May 2019.

29. Shankar N, Soe PM, Tam CC. Prevalence and risk of acquisition of methicillin-resistant Staphylococcus aureus among households: a systematic review. Int J Infect Dis. 2020;92:105-13.

30. Teo JW, Tan P, La MV, Krishnan P, Tee N, Koh TH, et al. Surveillance trends of carbapenem-resistant Enterobacteriaceae from Singapore, 2010-2013. J Glob Antimicrob Resist. 2014;2(2):99-102.

31. Teo JQ, Cai Y, Lim TP, Tan TT, Kwa AL. Carbapenem resistance in Gramnegative bacteria: the not-so-little problem in the Little Red Dot. Microorganisms. 2016;4(1):13.

32. Teo J, Ngan G, Balm M, Jureen R, Krishnan P, Lin R. Molecular characterization of NDM-1 producing Enterobacteriaceae isolates in Singapore hospitals. Western Pac Surveill Response J. 2012;3(1):19-24.

33. Koh TH. Gram-negative resistance in Singapore: a historical perspective. Ann Acad Med Singapore. 2008;37(10):847-54.

34. Navon-Venezia S, Kondratyeva K, Carattoli A. Klebsiella pneumoniae: a major worldwide source and shuttle for antibiotic resistance. FEMS Microbiol Rev. 2017:41(3):252-75

\section{Publisher's Note}

Springer Nature remains neutral with regard to jurisdictional claims in published maps and institutional affiliations.

Ready to submit your research? Choose BMC and benefit from:

- fast, convenient online submission

- thorough peer review by experienced researchers in your field

- rapid publication on acceptance

- support for research data, including large and complex data types

- gold Open Access which fosters wider collaboration and increased citations

- maximum visibility for your research: over $100 \mathrm{M}$ website views per year

At BMC, research is always in progress.

Learn more biomedcentral.com/submissions 